\title{
Research on Diesel Engine Piston Wear Fault Diagnosis Method Based on the Local Wave Time-frequency Analysis
}

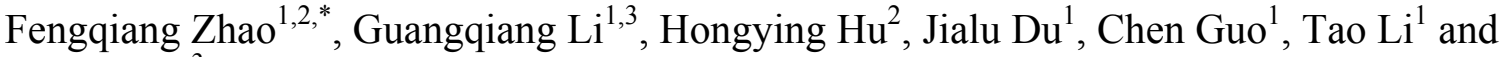 \\ Xinwen $\mathrm{Fu}^{3}$ \\ ${ }^{1}$ School of Information Science and Technology, Dalian Maritime University, Dalian 116026, China \\ ${ }^{2}$ College of Electromechanical \& Information Engineering, Dalian Nationalities University, Dalian 116600, China \\ ${ }^{3}$ Department of Computer Science, University of Massachusetts Lowell, Lowell, MA 01854, United States
}

\begin{abstract}
A large number of non-stationary dynamic signals are generated in the working machinery and equipment. Especially, diesel engines often encounter non-stationary transient and time-varying modulation signals, such as the impulse response signals caused by cylinder piston wear. These kinds of signals generated from diesel engine are analyzed by the method of the Local Wave time-frequency proposed in this paper, and then according to the analysis to diagnose the working state of the diesel engine. It proved that the proposed method is feasible and effective. Moreover, it provides an effective way for the diesel engine condition monitor and fault diagnosis.
\end{abstract}

Keywords: Fault diagnosis, Diesel, Local wave method, Time-frequency analysis.

\section{INTRODUCTION}

When Machinery and equipment have some fault, nonstationary transient and time-varying modulation signal are generated. For example, the frequency characteristics of these signals have relationship with their time, such as the impulse response of the cylinder piston wear signal which is generated from diesel engine, the signal generated when device starts or stops. Extraction and analysis the timevarying signals from diesel engine has a major significance to diesel engine fault diagnosis. Such signals must be analyzed in both time and frequency domains. Currently classical signal processing method based on Fourier transform is used commonly. The method only from the time domain or frequency domain gives the average results of the statistics of the signal, and it only takes into account the signal in time domain or frequency domain. The whole picture and the local variation in time domain and frequency domain cannot be considered at the same time. But the localized features are precisely the characterization of the fault. Local wave frequency is established based on the concept of instantaneous frequency. Moreover the instantaneous frequency is great significance to research on the phenomenon of transient and non-stationary. So it overcomes the limitations of traditional methods that it able to describe correctly the local features of the dynamic nonstationary signals in the time-frequency domain. In this paper, vibration signals generated from the impact on the cylinder of the piston in different wear conditions are studied by the local wave time-frequency analysis method. It proves by simulation and practical application that the local wave time-frequency analysis method is effective to diesel engine fault diagnosis.

\section{LOCAL WAVE METHOD}

In order to diagnose the working condition of the machinery and equipment, the signal acquired is usually expressed as a function of time or frequency, such as correlation analysis, power spectrum analysis and timefrequency analysis and so on. People choose different analysis methods based on different situations. However, these analysis methods give the energy of the same frequency signal within the analysis time or fixed time window, known as the full domain wave analysis. While impulse response signal is non- stationary and time-varying, which is caused by worn piston impacting cylinder, and the frequency of the signal may be only existed in the local time or instant of the signal course. The method used to analyse these kind of signals is called local wave analysis [1].

\subsection{The Principle of the Local Wave Method}

Local wave method is built on the concept of instantaneous frequency which is a very important physical quantity in the study of transient and non-stationary phenomenon $[2,3]$. The method takes the advantages of multi-resolution wavelet transform, at the same time overcomes the problems caused by different wavelet bases which are usually not self-adaptive and energy leakage of the signal. In order to get a meaningful instantaneous frequency, 


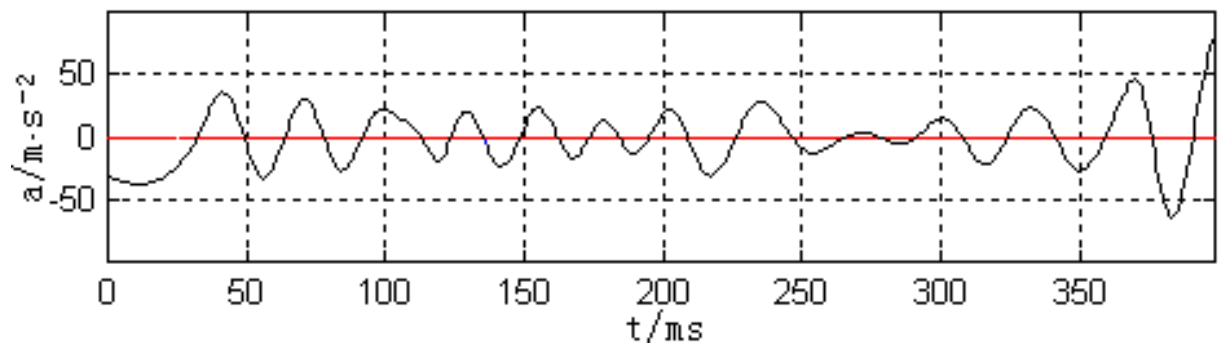

Fig. (1). One local wave component.

different decomposition algorithms $[4,5]$ can be used to obtain some finite local wave components, with these components, the instantaneous frequency of the time-varying signal and time-frequency characteristics will be able to be researched by the Hilbert transform. The local wave decomposition is a complete adaptive signal decomposition method. Due to the signal decomposition by the scale of the signal characteristics, the local wave decomposition is selfadaptive. With the instantaneous frequency, the signal can be analyzed by time-frequency domain. So the Analytical method to process signal is flexible and effective. In this paper, the local wave time-frequency method is proposed based on the local wave.

American scholar Huang has proposed an empirical mode decomposition method [6]. A series of intrinsic mode functions known as the local wave component can be obtained by the method. These components must meet the following qualifications:

(1) In the length of the data, the number of extreme points and the number of zero crossing must be equal, or at most a difference of one;

(2) At any time, the local maximum value of the signal and the local minimum definition of the mean envelope must be zero.

The first limited condition is similar to the traditional stationary Gaussian process defined on narrowband. The second qualification transforms the traditional global limit into the local limit, it can remove the fluctuations of the instantaneous frequency caused by the asymmetric waveform. In fact, the ideal situation is that the local mean is zero. But for non-stationary signals "local mean" involves "local time", it is difficult to be defined. Generally, the mean of the envelope is used as an approximation, which is called the local wave component. Because in each cycle defined by the zero points, there is only one basic pattern without complicated superposition of waves. A typical local wave component is shown in Fig. (1).

The corresponding analytic signal can be obtained by Hilbert transform of any component signal. Its Fourier transform is

$S(\omega)=\int_{-\infty}^{\infty} a(t) e^{j \theta(t)} e^{-j \omega t} d t=\int_{-\infty}^{\infty} a(t) e^{j(\theta(t)-\omega t)} d t$

According to the stationary phase principle [6], the frequency of the greatest contribution to $S(\omega)$ should meet the following limits. $\frac{d}{d t}(\theta(t)-\omega t)=0 \Rightarrow \omega=\frac{d \theta(t)}{d t}$

Obviously, we can obtain the expression which is accordant with the traditional definition of instantaneous frequency.

For a real signal, such as non-stationary signals, at some point there will certainly be more than one instantaneous frequency, which means that the data may contain more than one oscillation mode, so we must to effectively decompose signals, and then we can get a series of local wave components.

It should be emphasized that the decomposition is not for the purpose of the Hilbert transform, but for removing the superposition of local wave and local asymmetry and get meaningful instantaneous frequency. For each component, the instantaneous frequency can be defined everywhere. At the same time it should be pointed out that, due to the single component signal at any time has only one frequency, the frequency is called signal instantaneous frequency. Therefore, each component obtained through the local wave decomposition is the corresponding single components.

The process of decomposing complex non-stationary signals into a finite number of local wave components is called the local wave decomposition, also known as the shifting process. The corresponding decomposition algorithm is called local wave decomposition algorithm. Local wave decomposition algorithm flow chart is shown in Fig. (2).

If the local mean $m_{1}(t)$ is obtained from the original signal $S(t)$, in the ideal case, the first component is derived by following formula (3):

$$
h_{1}(t)=S(t)-m_{1}(t)
$$

Due to the approximation of the mean of the envelope, some non-symmetric waves may still exist in the $h_{1}(t)$, and therefore we need to continue to filter the $h_{1}(t)$, the process can be repeated several times, there is

$h_{11}(t)=h_{1}(t)-m_{11}(t)$

After $k$ times, we can get $h_{1(k-1)}(t)-m_{1 k}(t)=h_{1 k}(t)=C_{1}(t)$. 


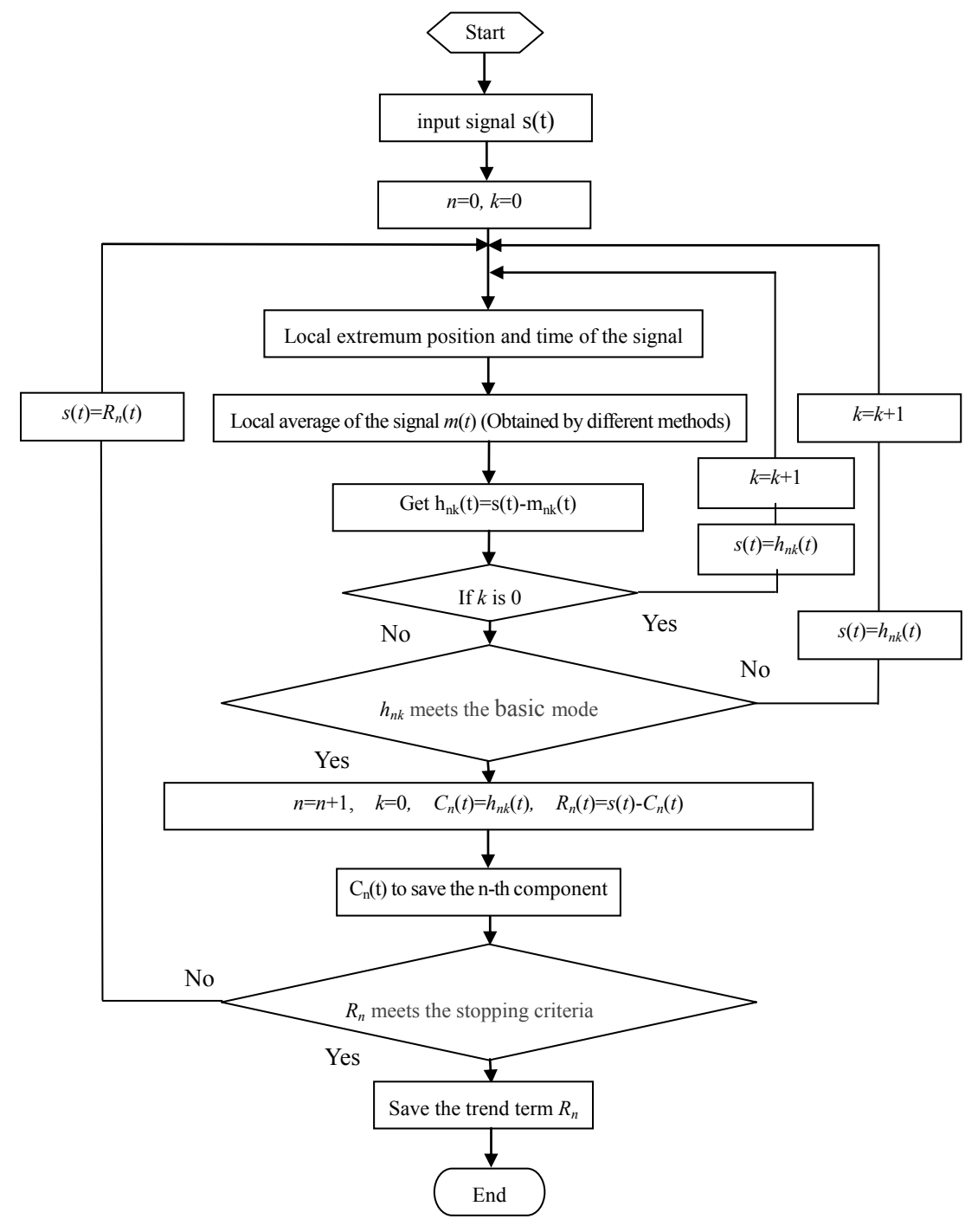

Fig. (2). Flow chart of local wave decomposition.

Where $C_{1}(t)$ is the first local wave components obtained from original signal. This process should be very careful, because too much repeat will lead local wave component into a purely frequency modulated signal and its amplitude becomes constant. To avoid this case, we must determine the criteria to stop the shifting process. The condition criterion can be achieved by limiting the size of the standard deviation. We can obtain the standard deviation $S_{d}$ by two consecutive processing in formula (5).

$$
S_{d}=\sum_{0}^{T}\left[\frac{\left|h_{1(k-1)}(t)-h_{1 k}(t)\right|^{2}}{h_{1 k}(t)^{2}}\right]
$$

Where $S_{d}$ can be selected between 0.2 and $0.3[4,6]$.

In general, the shortest cycle component of the original signal, i.e. the smallest part of the signal scales, should be included in $C_{1}(t) . R_{1}(t)$ is separated from the original signal $S(t)$, and processed as a new data.
$S(t)-C_{1}(t)=R_{1}(t)$

It is clearly, the large-scale part of the signal is contained in $R_{1}(t)$. We continue the shifting process in turn as follows.

$$
\begin{aligned}
& R_{1}(t)-C_{2}(t)=R_{2}(t) \\
& R_{2}(t)-C_{3}(t)=R_{3}(t) \\
& \quad \ldots \ldots \\
& R_{n-1}(t)-C_{n}(t)=R_{n}(t)
\end{aligned}
$$

Then there is

$$
S(t)=\sum_{i=1}^{n} C_{i}(t)+R_{n}(t)
$$

The original signal is decomposed into $n$-local wave component $\left(C_{1}(t) \sim C_{n}(t)\right)$ and a trend $R_{n}(t)$. Because the scale is increasing, so $R_{n}(t)$ is relatively slowly varying. It 
may be a monotonic function or a constant. Mode components $\left(C_{1}(t) \sim C_{n}(t)\right)$ contain the different scales information of the original signal after the separation.

To stop this process after the pre-conditions set criteria are met here stop conditions for the following categories.

(1) When the component $C_{n}(t)$ or the remaining component $R_{n}(t)$ becomes small enough to stop than the predetermined value.

(2) When the remaining component $R_{n}(t)$ becomes a monotonic function, which can no longer filter out the local wave component so far. Even if the original signal data with global zero mean, the last of the remaining amount may still not be zero. For the data with the trend term, the remaining component is the trend term.

It can be seen from this process, each component can be amplitude or frequency modulation $[4,6]$. Through the decomposition, different amplitude and frequency modulation is also clearly separated. We obtain a signal description method of variable amplitude and variable frequency. Decomposition can effectively separate the different time scales, and deal with non-stationary, nonlinear and localize time-frequency at the same time. The method of decomposition has a strong adaptability.

\subsection{Time-Frequency Spectrum of Local Wave}

We can obtain limited number of components by signal decomposed by the local wave method, then change the component signals $C_{i}(t)$ into analytic signal by Hilbert transform.

$$
Z_{C_{i}}(t)=X_{C_{i}}(t)+j Y_{C_{i}}(t)=a_{C_{i}}(t) e^{j \phi_{C_{i}}(t)}=a_{C_{i}}(t) e^{j \omega_{C_{i}}(t) t}
$$

Where

$$
a_{C_{i}}(t)=\left[X_{C_{i}}(t)^{2}+Y_{C_{i}}(t)^{2}\right]^{\frac{1}{2}}, \quad \phi_{C_{i}}(t)=\arctan \frac{Y_{C_{i}}(t)}{X_{C_{i}}(t)}
$$

Then, we can gain $\omega_{C_{i}}$ which is the instantaneous frequency of the component.

$\omega_{C_{i}}=\phi_{C_{i}}^{\prime}$

Component signal $C_{i}(t)$ can be expressed as follow. $C_{i}(t)=\operatorname{Re}\left[a_{C_{i}}(t) \exp \left(j \int \omega_{\mathrm{C}_{\mathrm{i}}}(\mathrm{t}) \mathrm{dt}\right)\right]$

Where $\mathrm{Re}$ is the real part, then there exists

$$
s(t)=\operatorname{Re}\left[\sum_{i=1}^{n} a_{C_{i}}(t) \exp \left(j \int \omega_{C_{i}(t)} d t\right)\right]
$$

Where $n$ is the number of components. The residual trend component can be treated as a long period of low frequency vibration by Hilbert transform. The component is a small offset in the energy. Taking into account the uncertainty of the long trend component, and in order to better observe the local wave component which contains useful low-frequency information, the residual amount usually is not considered during the Hilbert transform in this paper.

Using the Fourier expansion the same data, $s(t)$ is

$s(t)=\operatorname{Re}\left[\sum_{i=1}^{\infty} a_{i} e^{j \omega_{t} t}\right]$

Where $a_{i}$ and $\omega_{i}$ are constant. The contrast is clear in formulas (10) and (11). Each local wave component describes a generalized Fourier changes. The variable magnitude and the instantaneous frequency are not only greatly improved the efficiency of expanding the signal, but this decomposition can handle non-stationary data.

As a result, the limitations of the Fourier expansion of fixed amplitude and fixed frequency are broken, and the method of description variable amplitude and variable frequency signal is acquired.

Signal amplitude can be expressed as a function $H(\omega, t)$ of time and frequency in the three-dimensional space by the formula (10) which is called the local wave time-frequency representation of the signal in this paper. It also be referred to as the local wave time-frequency spectrum which is expressed as follow $[1,4,7]$.

$$
H(\omega, t)=\operatorname{Re}\left[\sum_{i=1}^{n} b a_{C_{i}}(t) \exp \left(j \int \omega_{C_{i}(t)} d t\right)\right]
$$

Where $\mathrm{n}$ is component number. When $\omega_{C_{i}}(t)=\omega$, the variable $b=1$, otherwise $b=0$. According to the need, timefrequency spectrum of the component can also be obtained.

$$
H_{C_{i}}(\omega, t)=\operatorname{Re}\left[b a_{C_{i}}(t) \exp \left(j \int \omega_{C_{i}(t)} d t\right)\right]
$$

Or the reconstruction time-frequency spectrum of several components can be gained.

$$
\begin{aligned}
& H_{C_{i} \cdots C_{k}}(\omega, t)=\operatorname{Re}\left[b a_{C_{i}}(t) \exp \left(j \int \omega_{C_{i}(t)} d t\right)\right] \\
& +\cdots+\operatorname{Re}\left[b a_{C_{k}}(t) \exp \left(j \int \omega_{C_{k}(t)} d t\right)\right]
\end{aligned}
$$

The amplitude of the signal can be expressed as contour lines on the time-frequency plane, or three-dimensional map in such a three-dimensional plane $\{t, \omega, H(\omega, t)\}$.

\section{DIAGNOSIS EXAMPLE}

In a large number of components of a diesel engine, the wear of the diesel engine cylinder liner and piston is one of the reasons a direct impact on the performance of diesel engine. The fault diagnostic criterion of the wear between the cylinder liner and piston acquired by a large number of 


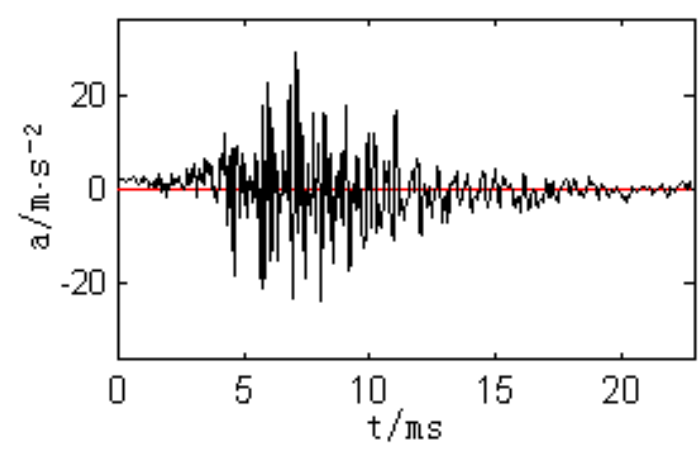

A. Normal peak signal

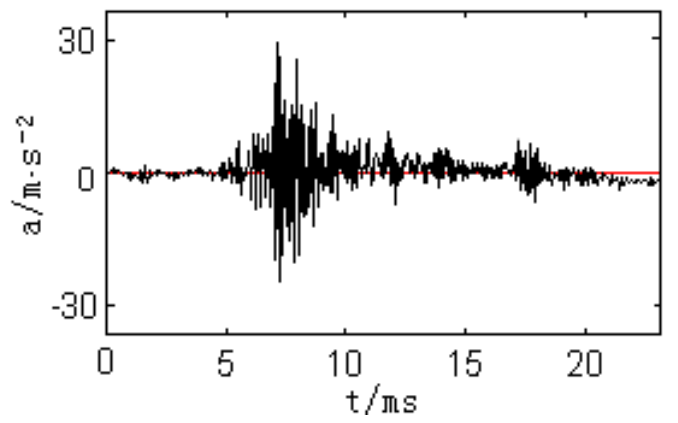

B. Slightly wearing signal

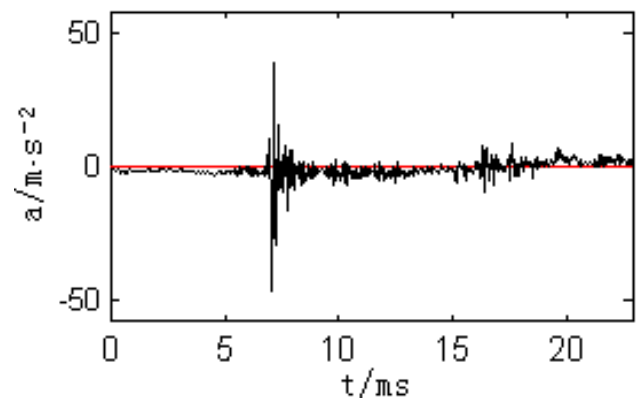

C. Serious wearing signal

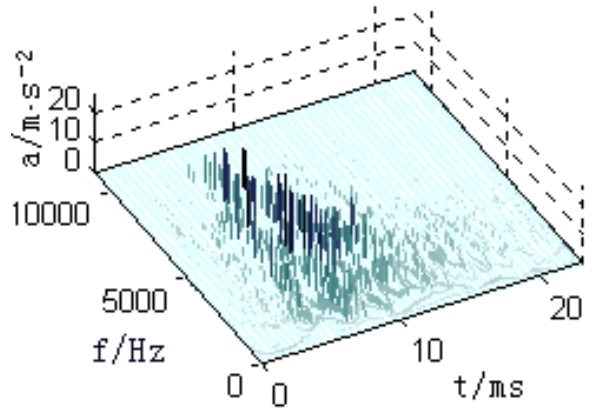

D. Time-frequency spectrum of normal signal

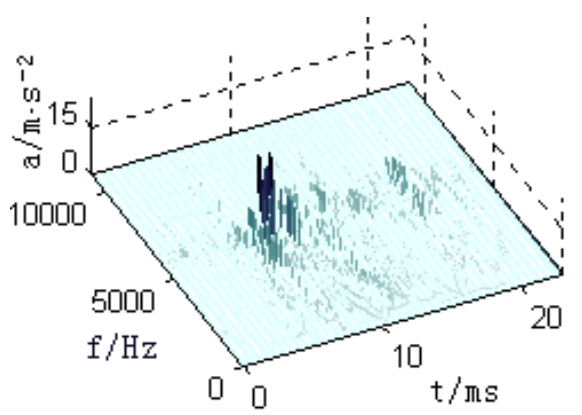

E. Time-frequency spectrum of slightly wearing signal

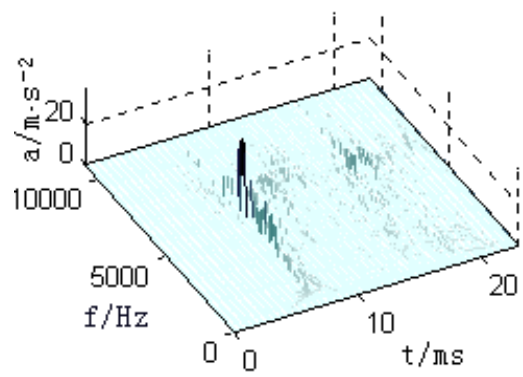

F. Time-frequency spectrum of serious wearing signal

Fig. (3). Vibration signal of the cylinder head and corresponding time-frequency spectrum (A-F).

simulation experiments is given in reference [8]. This Experimental study is important, but because of the differences in laboratory and field work environment and external uncertainties, testing and analysis results in the laboratory are often difference from in the field. Wear mechanism is researched in the practical application in reference [9], but it is difficult to make a valid judgment to the degree of wear. While the method of the time-frequency analysis can correctly describe the local characteristics of the dynamic non-stationary signals in the time-frequency domain, and can to reveal the composition of the frequency, distribution and arrangement of the vibration signal of each band in the local time. In this paper, the method of the timefrequency analysis of the local wave is used to diagnose failure of the field devices. Diagnosis example is given as follows.
The cylinder head vibration signal is measured in the same position under the normal state of the diesel engine, a piston slight wear state and serious wear condition. Where analytical frequency of vibration signal is $10 \mathrm{kHz}$, sampling frequency is $25.6 \mathrm{kHz}$, and the engine speed is $710 \mathrm{r} / \mathrm{min}$.

When the diesel engine cylinder liner piston worn, the impact of the piston on the cylinder liner is mainly reflected in the cylinder pressure signal of the outbreak period, according to the characteristics of this signal, the degree of wear of the cylinder liner piston can be determined. Therefore the signal which is intercepted in the expansion stroke near the top dead is analyzed in the A to C of Fig. (3). Vibration waveform can be seen that there are some differences from the time domain waveform. The amplitude increasing gradually that indicate the vibration strengthen, but slightly worn state and normal state signal is not very 
different. Time-domain waveform cannot give the characteristics of the signal frequency. The corresponding time-frequency spectrum is given in the D to F of Fig. (3). It is clearly seen that vibration signal mostly concentrated in the frequency of 3000 to $6000 \mathrm{~Hz}$ nearby for the energy distribution of the normal signal. The vibration signal has certain duration. It is mainly concentrated in the 5 to $12 \mathrm{~ms}$ time period that includes the energy of the low frequency part. When fault occurs, the clearance between the piston and cylinder is enlarged and the speed of the piston impacting cylinder liner will change which results in impact energy. At the same time, due to the gap changes, the lateral movement of the piston in the cylinder changes. Then the number and the location that the piston hits the liner changes, which will cause vibration characteristics of the liner change [10]. Minor wear, the energy of the vibration signal enlarge. The energy in the high frequency is significantly greater than that in the low frequency and the duration is relatively reduced. There is still significant vibration in the time period of 18 to $20 \mathrm{~ms}$. During severe wear, energy are prominent only in the range of $7000 \mathrm{~Hz}$ to $8000 \mathrm{~Hz}$ in the time-frequency spectrum. At the same time, the duration decreases rapidly, only about $2 m s$ or so.

\section{CONCLUSION}

The local wave decomposition is an adaptive basis functions mode decomposition method. The method can handle non-stationary and nonlinear signal and has selfadaptability. It can control the pollution of the singular signal to a small range. The signal at the point singularity can only influence local, but will not spread to the entire interval. Local wave frequency analysis method is a generalized linear time-frequency representation, since they are built on the basis of the instantaneous frequency, which is different from the definition of Fourier analysis in the frequency. Due to the localization of time domain and frequency domain, Local wave analysis method is different from the others signal decomposition method and time-frequency analysis. In this paper, we verify that the application of local wave frequency analysis is feasibility by the analysis characteristics of the vibration signal of diesel engine cylinder head. For piston wear failure of the diesel engine cylinder liner, we comprehensively analyze the vibration signal of the cylinder head by the local wave frequency analysis method. Then we can identify the fault. At the same time, it will reflect the failure level. It demonstrates that the method is effective to analyze the vibration characteristics of reciprocating machinery and extract fault information. This method has great engineering application value. So it is verified that the local wave analysis method is effective for fault diagnosis of reciprocating equipment.

\section{CONFLICT OF INTEREST}

The author confirms that this article content has no conflict of interest.

\section{ACKNOWLEDGEMENTS}

We would like to express our gratitude to the National Natural Science Foundation of China (No. 51079013, No. 61074053 and No. 61374114), the Fundamental Research Funds for the Central Universities of China (No. DMU2011NQ030, No. DC120101014, No. DC110320), the Applied Basic Research Program of Ministry of Transport of China (No. 2011-329-225-390, No. 2012-329-225-070), the China Scholarship council (No. 201306575010), and the Higher Education Research Fund of Education Department of Liaoning Province of China (No. LT2010013) for financial support of our work.

\section{REFERENCES}

[1] X. Ma, B. Yu and Y. Cai, "A new time-frequency analysis method - local wave method", Journal of Vibration Engineering, vol. 13(S), pp. 219-224, 2000

[2] Y. Liu, Y. Tan, H. Xie, W. Wang and Z. Gao, "Time-frequency analysis of non-stationary fusion plasma signals using an improved Hilbert-Huang transform", Review of Scientific Instruments, vol. 85, no. 7, pp. 3502, 2014.

[3] I. Orovic, A. Draganic, S. Stankovic, and E. Sejdic, "A unified approach for the estimation of instantaneous frequency and its derivatives for non-stationary signals analysis", In 11th International Conference on Information Science, Signal Processing and their Applications, pp. 1130-1134, 2012.

[4] Y. Zou, "Research and Application on the Theory and Method of Local Wave Analysis", Dalian: Dalian University of Technology, 2004.

[5] Q. Gai, X. Ma, H. Zhang, "Comparing study for decomposing methods using local wave method", Systems Engineering and Electronics, vol. 24, no. 2, pp. 57-59, 2002.

[6] N.E. Huang, Z. Shen, S.R. long, M.C. Wu, H.H. Shih, Q. Zheng, N. Yen, C.C. Tung and H.H. Liu, "The empirical mode decomposition and the hilbert spectrum for nonlinear non-stationary time series analysis", Proceedings A Royal Society London, vol. 471, no. 2175, pp. 903-995, 1998.

[7] B. Yu, "Adaptive Time-Frequency Analysis Method and its Applications in Fault Diagnosis", Dalian University of Technology, Dalian, 1998.

[8] B.L. Song, "The research on fault diagnosis of diesel engine based on EMD”, Applied Mechanics and Materials, vol. 623, pp. 143148, 2014.

[9] X. Wang, C. Liu, F. Bi, X. Bi, and K. Shao, "Fault diagnosis of diesel engine based on adaptive wavelet packets and EEMD-fractal dimension", Mechanical Systems and Signal Processing, vol. 41, no. 1-2, pp. 581-597, 2013.

[10] L. Feng and F. Feng, "Wavelet transform technology in the nonstationary signal fault diagnosis in engineering application", Advanced Materials Research, vol. 518-523, pp.1355-1358, 2012.

Received: September 22, 2014

Revised: November 03, 2014

Accepted: November 06, 2014

(C) Zhao et al.; Licensee Bentham Open.

This is an open access article licensed under the terms of the Creative Commons Attribution Non-Commercial License (http://creativecommons.org/licenses/by-nc/3.0/) which permits unrestricted, non-commercial use, distribution and reproduction in any medium, provided the work is properly cited. 